\title{
System for Automated Infrared Image Processing Based on Neural Network Technologies
}

\author{
Sergey S. Kananadze \\ Moscow Aviation Institute (MAI), Volokolamskoe Highway, 4, 125993, Moscow, Russia
}

\begin{abstract}
The article describes the main problems that non-destructive control experts encounter in processing and analyzing infrared (IR) images obtained using thermographic cameras: noisiness, information redundancy, excessive data volume, etc.

A gradual process of automated digital processing and analysis of images on the basis of a software system is considered, which allows not only overcoming the drawbacks of the raw data described above, but also drawing a conclusion about the state of the object based on the obtained image independently. The ability to make expert decision is provided by a neural network component in a system on the basis of Kohonen network used for cluster image analysis. There are the examples of system operation applied to IR images of the lining of solid domestic waste (SDW) incinerator.

The use of such systems allows reducing workload of an expert in processing large volumes of data and reducing the probability of errors due to such effect as vision "blurring".
\end{abstract}

Keywords - cluster analysis, digital processing, image processing, NN, thermographic camera.

\section{Introduction}

Currently, in industrial enterprises and during the production, more and more attention is paid to nondestructive control and diagnostic methods.

DOI: 10.18421/TEM94-16

https://doi.org/10.18421/TEM94-16

Corresponding author: Sergey S. Kananadze, Moscow Aviation Institute (MAI), Moscow, Russia.

Email: kananadze@yandex.ru

Received: 09 June 2020.

Revised: 26 October 2020.

Accepted: 30 October 2020.

Published: 27 November 2020.

(c))BY-NC-ND (C) 2020 Sergey S. Kananadze; published by UIKTEN. This work is licensed under the Creative Commons Attribution-NonCommercial-NoDerivs 4.0 License.

The article is published with Open Access at www.temjournal.com
One of the widespread methods is control with the use of IR devices, which, in particular, include thermographic cameras.

It should be noted that in some cases thermocouples (thermoresponsive sensors) are used to measure temperature. The advantage of such devices over other temperature recorders (thermographic cameras, in particular) is that they calculate the actual and not the effective temperature of the object. However, their major disadvantage is that they cannot give a holistic thermal picture for the entire object of study.

The widespread use of thermographic cameras as one of the means of non-destructive control and diagnostics of products of various profiles is due to the fact that they allow diagnosing the object condition without direct contact with the object. These devices allow obtaining the thermal field of the object in the form of an IR image, i.e. they convert the object's thermal radiation invisible for the naked eye into the image on the display.

There is the number of typical tasks solved with the help of thermal imagers [1]:

- detection - establishing the presence of a radiating object in the corner field of a thermal imaging system (TIS) or on a display screen (display system);

- classification - detected object belongs to some fairly wide class, for example, to aircraft, etc.;

- recognition - establishing the belonging of an object to a relatively narrow class, for example, that the object is an airplane, etc.;

- identification - determination of a specific type of object, for example, an airplane model, etc.

Sometimes the totality of all these tasks is called detection and recognition.

Fast response time, ability to operate at any time of the day, accuracy, and lack of contact with the object during the measurement process allow effective use of thermographic cameras in industry, construction, aviation, etc.

Thermographic cameras allow obtaining image of the thermal field of the object, in which the hottest areas correspond to the bright areas in the image, and the 
coldest areas correspond to the dark ones. In this regard, the recorded temperature is effective, i.e. the radiation intensity of the object corresponding to this temperature corresponds to the radiation intensity of an absolutely black body at the same temperature [1]. If there is information about the emissivity of the object, it is possible to obtain the value of its actual temperature. When using thermovision systems, it should be taken into account that thermal radiation is weakened due to absorption and scattering by gas molecules in the air.

The modern process of processing, analysis, and classification (PAC) of IR images can be represented as follows: Object -> [Thermal imager, System of computational IR thermography (SCIRT), Thermal Sensors] -> IR Image -> Expert -> Result.

The specialist observer analyzes the IR image and forms a certain expert opinion on the basis of the available information about the object. The SDW incinerator lining was taken as the object of the study (Figure 1.). The problem of waste disposal is quite acute in the modern world, and the equipment responsible for its disposal should be in good condition. Timely and high-quality diagnostics will allow avoiding labor-intensive and expensive repair of the furnace with the complete replacement of the lining. Otherwise, waste disposal enterprise may incur significant losses.

Thus, the expert opinion on the incinerator lining should include information, for instance, about its condition, the presence of burnouts in it, and the need for repair.

The expert presented in this chain generates a conclusion on the object, based on the obtained data, goals, and objectives of the study. However, any specialist can give only a subjective conclusion. In addition, the observer generates his assessment, using in most cases an unprocessed IR image, in addition, when processing and analyzing large amounts of information, the quality of decisions made (their reliability) will fall over the time of the expert's work, and here the computer will be simply irreplaceable.

Therefore, we can conclude that the main drawback inherent in the above process of PAC IR images is the presence of an expert. Therefore, the problem of automation of this process is very relevant. In this regard, in various works a lot of attention was paid, paid and will be paid to the methodology of automation of obtaining and processing IR data of objects of research, the history of the formation and development of which we will consider below.

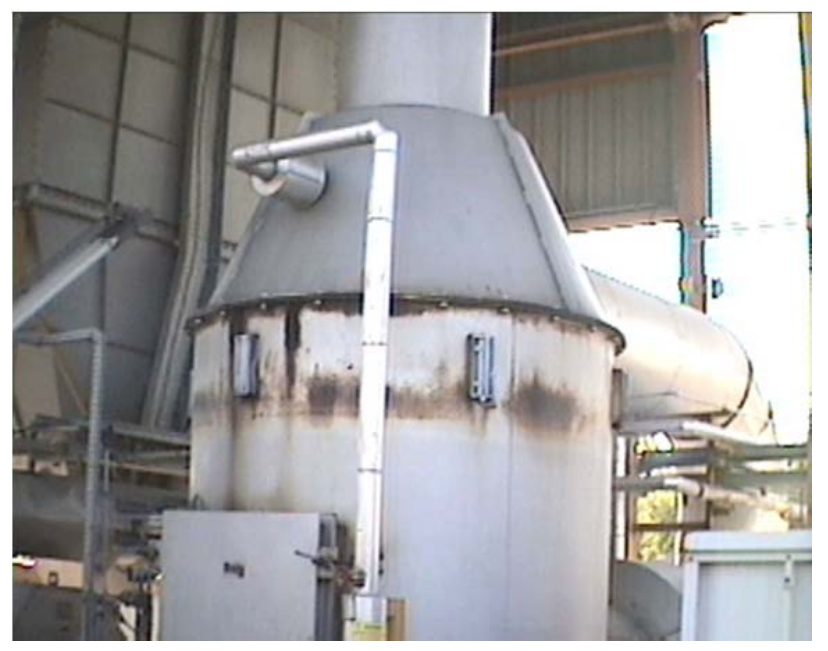

Figure 1. SDW incinerator

\section{Review of Research in the Field of Processing IR Data Obtained Using Thermal Imaging Technology; Literature Review}

The beginning of the development of infrared (IR) thermography in the USSR in 1972 was started by academician of the RAMS V.P. Kaznacheev, and his work was continued by the staff of the Institute of Radio Engineering and Electronics of the Russian Academy of Sciences, Academician Yu.V. Gulyaev and Professor E.E. Godik. They were interested in man as a biological object that generates electromagnetic waves. Almost at the same time, in large scientific research institutes of the country, work began on the creation of devices (thermal imagers) that allow contactless recording of thermal radiation from any object (not necessarily living) [2].

The consistent development of computational thermophysics, thermometry, and the theoretical foundations of non-contact diagnostics of complex systems according to temperature characteristics and the improvement of infrared optical systems have largely determined the formation of a new scientific direction: computational infrared thermography.

The first SCIRT appeared in 1990. These systems, designed to restore and visualize temperature at any point in the object (including unobservable), use numerical models of processes based on a priori information about the parameters of the object and the results of the intensity of infrared radiation object [3], [4].

Traditional IR image processing systems restore the distribution of temperature fields on the surface of an object using a rigid algorithm that does not always give the correct results [3]. SCIRT, unlike traditional systems, takes into account the selective nature of the sensitivity of sensors, absorption in the atmosphere and IR filters, ambient temperature and its effective emissivity. 
The SCIRT complex includes:

a) software and metrological tools;

b) equipment for inputting information about the spatial and spectral distribution of IR radiation intensity and temperature of objects (thermal imager, IR spectrophotometer, multichannel thermocouple analyzer, and IR photodiode sensors);

c) programmable means of digital processing and displaying information according to specified algorithms.

The general structure of SCIRT is shown in the Figure 2.

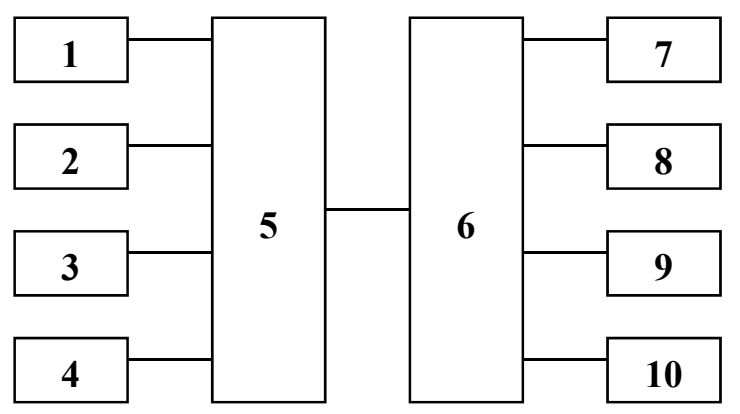

1 - powder dosing and feeding system, 2 - mixing chamber with nozzle block, 3 - control system for parameters and mass flow of carrier gas, 3' - powder dispenser control

system, 4 - carrier gas heater, 5 - heterogeneous supersonic flow, 6 -work piece, 7 - work piece movement mechanism, 8 - compressed air cylinder

Figure 2. General view of the SCIRT structure

The programs used in the SCIRT complex provide adaptive control of the experiment and data input, correction and processing of IR images, modelling of the studied physical processes, graphical display of results of the experiments, and numerical modelling.

Although SCIRT solves the problems of efficiency and automated processing of received data, the question of the integrity of the information received about the object remains unresolved [1]. High-quality thermal imagers had a high price, therefore, basically, thermocouples were used in SCIRT, which led to the above-described information integrity problem. In addition, SCIRTs were bulky, and their portability left much to be desired.

Current problems of physical research and nondestructive testing required the creation of image converters in a wide range of wavelengths: from $\mathrm{x}-$ rays to radio waves. With the development of science and technology, the appearance of such converters thermal imagers - devices that create and convert infrared (IR) radiation of heated bodies into visible and thus provide visualization of the thermal field of heated objects has become possible [1], [3]. They incorporated all the advantages of SCIRT in the field of automated processing and data acquisition efficiency, and at the same time got rid of the disadvantages of these systems.

To obtain a thermal image, infrared (IR) radiation is used, the source of which is the object. Detailed expositions of the laws of radiation of heated bodies and the laws governing the formation of IR images are given in special monographs [5] and in works devoted to optoelectronic IR devices [3], [6].

In the USSR, work on the creation of such devices was laid by the director of the Institute of Radio Engineering and Electronics Yu.V. Gulyaev and continued by his followers: Director of the small enterprise "IRTIS" at the Research Center for Electronic Diagnostic Systems of the Russian Academy of Sciences M.I. Shcherbakov and employees of the company "IRTIS" M.G. Utkin, D.V. Spirin, N.V. Kozhakin and N.V. Zimokha. Their efforts created the IRTIS-200 and IRTIS-2000 thermal imagers.

From the moment of the appearance of thermal imagers, up to the present day, developments are constantly underway to improve the electronic block of thermal imagers, which is responsible for converting a thermal image into an electrical signal [1], [7]. Examples of devices that make up such a unit include a thermoelectric converter (developed and patented by V.D. Bobryshev and A.K. Blazhis), and a multi-element photo detector with a longwavelength absorption limit of up to $7 \mu \mathrm{m}$ (developed and patented by A.V.Dvurechensky , A.P. Kovchavtsev, G.L. Kuryshev, I.A. Ryazantsev, patent holder, Institute of Semiconductor Physics, Siberian Branch of the Russian Academy of Sciences).

Currently, computers are also being delivered together with thermal imagers with specialized software systems, which include the following methods for analyzing thermograms (IR images):

a) measuring the absolute temperature at a point or zone;

b) building a thermal profile in any position;

c) construction of an isotherm;

d) plotting the temperature changes over time (for a dynamic thermographic film);

e) listing of thermograms and reports.

Also, these complexes include functions for processing images of thermograms to improve their visual perception: smoothing, sharpening, contrasting, cropping, rotation, color correction. An example of this specialized computing complex is the IRTIS software package developed at the "IRTIS" small enterprise.

The thermal imager software driver allows performing functions, some of which were previously assigned to computers in SCIRT: 
- providing visualization of thermal images in real time;

- output of maximum, minimum, and average temperatures in the frame field;

- frame stop mode for preliminary analysis;

- recording individual thermograms;

- ability to record dynamic thermographic film;

- temperature measurement mode;

- auto-tuning of the dynamic range;

- accounting for reflection coefficient;

- ability to select different color palettes and isotherms;

- frame-by-frame summation mode.

The set of "thermal imager-computer-printer" has become an analogue of SCIRT, used earlier, but at a qualitatively new level. However, in some cases, the software and computer systems do not independently analyze the received IR images, but only prepare them, conducting preliminary processing, for analysis by an expert.

In the digital analysis of IR images associated with the processing and analysis of large amounts of information, a specialized computing system comes to the aid of an expert, which allows him to be almost completely eliminated from this process, taking on the main work. The integration of these two stages for processing and analyzing large arrays of digital information, which are sets of IR images, is expressed in the creation of a specialized computing system that allows the expert to be almost completely excluded from this process, taking on the main work. Functionally developed system for automated processing and analysis of IR images is a set of blocks, each of which is responsible for solving a specific task during the program. Blocks are combined into modules for their intended purpose, and each of them is responsible for a specific stage in IR image processing. The main methods that formed the basis for the functioning of this system are discussed in the next two sections.

\section{Theoretical Basis}

Structural scheme of the thermographic camera corresponding to the general scheme of its operation is shown in the Figure 3. It shows only the main links involved in the conversion of the optical signal that characterizes object space in the invisible (IR) spectrum region into a visible image perceived by the observer. The highlighted links belong to the thermographic camera.

As it is shown in the Figure 3., the main elements of the thermographic camera, which make it different from the other optical systems, are radiation receiver, electronic processing chain, and display. The thermal (invisible) radiation received from the object enters the radiation receiver, then it is processed in the electronic processing chain, and then it is put on the display as an IR image of the thermal field of the object.

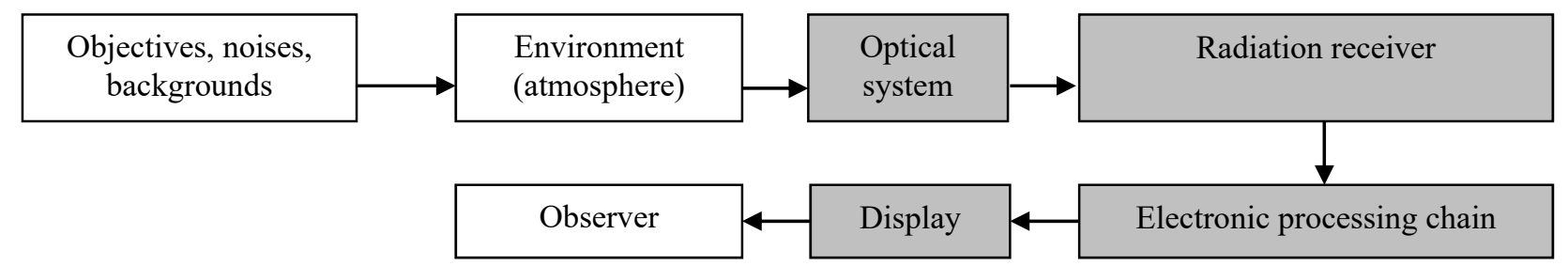

Figure 3. Scheme of signal conversion by means of thermographic camera

To achieve the goal-automation of the PAC process of IR images, the author proposes to gradually solve the tasks inherent in many systems of the same class.

The signal (radiation) from the object, passing through the atmosphere and the optical system of the thermal imager, is exposed to noise superimposed on the original signal by suspensions and atmospheric turbulence, weather events, and hardware interference in the thermal imager. Therefore, firstly, preliminary processing of IR images is required, since the analysis of the "raw" picture can distort the final result and lead to an incorrect conclusion about the object. When pre-processing IR images, mathematical methods are used that have long and successfully established in the field of image processing: median filtering, threshold segmentation, pyramidal representation, etc. It should also be noted that these methods are moderately demanding on the hardware of the computer, which expands the scope of their possible applications.

Median filtration of the image is performed by the following formula [8]:

$$
g(m, n)=\operatorname{med}\{f(x, y)\},(x, y) \in W(m, n),
$$

where $W(m, n)$ is the region with the centre at the image point with coordinates $(m, n)$, from the samples of which the median $g(m, n)$ at the point $(m, n)$ is calculated; $f(x, y)$ is the brightness function of the image point with coordinates $(x, y)$.

The function med\{.\} from a set of $\mathrm{N}$ values is determined as follows: it sorts the values from the given set in ascending order and selects the value with the number $((\mathrm{N}-1) / 2)+1)$ from the sorted set. If 
$\mathrm{N}$ is even, the value obtained as the as the arithmetic mean of the values from the sorted set with numbers $(\mathrm{N} / 2)$ and $((\mathrm{N} / 2)+1)$ can serve as "averaging" value of the set.

The most commonly used square mask is of size $(2 k+1) \times(2 k+1), k=1,2, \ldots$, and at each point of raster $(i, j)$ brightness is recalculated by the following rule: the mask is placed so that its center coincides with point $(i, j)$, and the brightness $(2 k+1)^{2}$ of the image elements that get into the mask are numbered in ascending (descending) order: $b_{1} \leq b_{2} \leq \cdots \leq b_{i}$. The set $b_{1}, \ldots, b_{i}$ may contain equal values numbered by different indices. The middle element of the set $b_{1}, \ldots, b_{i}$ will be its median.

Threshold image processing means the conversion of its brightness function by the operator $\mathrm{T}: \mathrm{f}(\mathrm{x}, \mathrm{y}) \rightarrow$ $\mathrm{S}(\mathrm{x}, \mathrm{y})$,

$$
S(x, y)=\left\{\begin{array}{l}
\lambda_{j}, \text { if } T_{j} \leq f(x, y)<T_{j+1}, \\
\lambda_{0}, \text { if } f(x, y)<T_{0} \\
\lambda_{k-1}, \text { if } f(x, y)>T_{k-1}
\end{array}\right.
$$

where $\mathrm{f}(\mathrm{x}, \mathrm{y})$ is the brightness function of the image; $\mathrm{S}(\mathrm{x}, \mathrm{y})$ is the segmented image; $\mathrm{k}$ is the number of segmentation regions; $\lambda_{0}, \ldots, \lambda_{k-1}$ are the names of segmentation regions; $T_{0}, \ldots, T_{k-1}$ are threshold values, arranged so that $T_{0}<\cdots<T_{k-1}$. Points of the original image, the brightness of which lie in one interval, form a homogeneity class identified by the mark $\lambda_{i}, i=\overline{0, k-1}$.

In the pyramidal way of representing images, a pyramid [9] is used, which is a hierarchical set of brightness matrices of the same image, in which the first matrix is the original image with the maximum resolution, and each subsequent matrix is the same image, but with a lower resolution (this is usually a row of degree number 2). A schematic view of the pyramidal method of presenting images is shown in the Figure 6 . The type of performed operation is local.

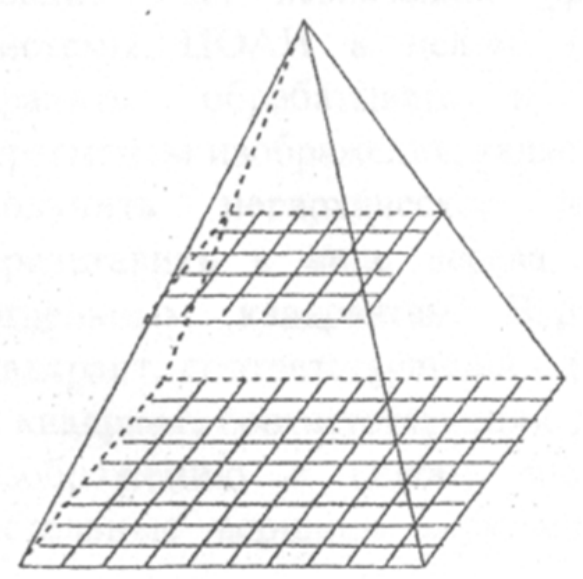

Figure 4. Pyramidal method of presenting images
To highlight informative areas in the image (for example, for some further research), the "bug" algorithm is used. In the general case of a black and white image, bug starts moving from the white region towards the black one. As soon as it gets on the black element, it turns left and moves on to the next element. If this element is white, the bug turns right, otherwise it turns left. The procedure keeps repeating until the bug returns to the initial point. The coordinates of the transition points from black to white and from white to black describe the boundaries of the object. In this case, the last point of the object, and not the first point of the background is marked as the boundary of the object (contour) [10].

Secondly, it is necessary to choose a method that will be used in the analysis and subsequent classification of the processed IR image. This stage should be given special attention, as it is at this stage that an analytical decision is made, which is characteristic of a person, and an "expert" opinion on the object is formed. The word "expert" is in quotation marks, because in this case, the computer acts as an expert, and not a person. One of the modern approaches to the analysis and classification of IR images in the conditions of an unknown number of cluster groups, allowing taking into account the features of this stage, is cluster analysis, which formed the basis of the system at this stage.

For cluster analysis, the Kohonen network is often used, which belongs to the class of fully connected networks and consists of $\mathrm{M}$ neurons that form a rectangular lattice (or ruler) on the plane. In the network, formal ("classical") neurons, which are linear weighted adders, are used as neurons. Their output is formed by the following formula:

$$
Y=F\left(\sum_{i} w_{i} x_{i}\right)
$$

where $Y$ is the neuron output, $w_{i}$ is the weight coefficient of the i-th input, $x_{i}$ is the value at the i-th input (i-th component of the input vector), $F$ is the activation function of the neuron (usually nonlinear). The Kohonen network has two layers: input and output. The number of neurons in the output layer usually coincides with the number of proposed classes into which the input images will be divided. However, it is permissible to use a larger number of neurons in order to reflect intermediate states of belonging to classes. All inputs are associated with all output neurons. There are no feedbacks. Input signals, vectors of real numbers, are sequentially presented to the network. Desired output signals are not determined. During the operation of the algorithm, the synaptic weights of the neurons are tuned. After a sufficient number of input vectors have been presented, the synaptic weights of the network are determined by the clusters. In addition, weights are organized so that topologically close nodes are sensitive to similar external influences (input signals). 
The advantages of this network are that, for example, in contrast to the Grossberg Adaptive resonance theory network [9], the Kohonen network is able to function in the conditions of interference inherent in IR images, since the number of classes is fixed, the weights are modified slowly, the adjustment of weights ends after training (in Adaptive resonance theory network configuration continues uninterrupted).

Moreover, the Kohonen network, which is trained without a teacher, is invariant to rotations, displacements, and shapes of critical areas in IR images, which reduces the number of training samples (thereby increasing the speed of network learning) and makes the network learning process logically transparent.

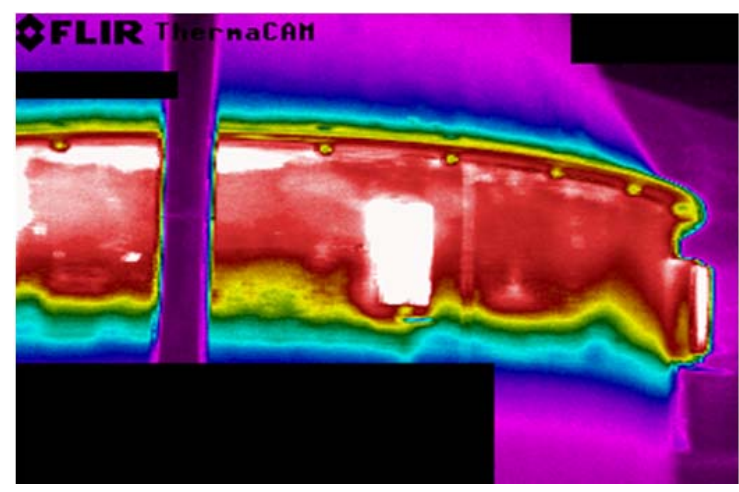

Figure 5. IR images of the incinerator lining from different positions
Figure 6. shows in general terms a modular-block scheme of the functioning of the software system developed by author. It is shown in the Figure 6., that operation of the system is divided into 2 stages: preprocessing of images, consisting of a set of sequentially working blocks, such as "Filtration", "Segmentation", "Reduction", "Contouring", and the cluster analysis, which is responsible for decision forming on the state of the object. The cluster analysis module receives IR image pre-processed by module № 1 .

Algorithmic software of the system was developed in the $\mathrm{C}++$ programming language in the Integrated Development Environment C ++ Builder (IDE). This environment is convenient for the quick development of multi-window applications with numerous graphs

\section{Methodology}

To obtain the initial IR images for processing, an investigation of the lining of the SDW incinerator shown in the Figure 1. was carried out at the incinerator, using the FLIR Thermacam thermal imager. The study was conducted under conditions corresponding to the operating parameters of the thermal imager with a small content of extraneous suspensions in the atmosphere. Examples of obtained images are presented in the Figure 5. For highquality display of survey results, the raster type was selected for representing IR images in the form of a two-dimensional matrix of pixels of size $320 \times 240$ on the basis of a full-color 24-bit RGB model [11].

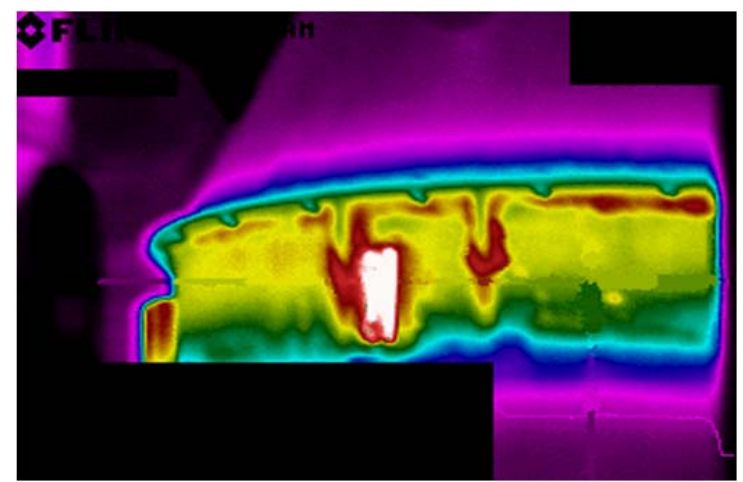

and figures, which allows combining computational power of the $\mathrm{C}++$ language and the convenience of development of an interface using Rapid Application Development (RAD) technology [12]. The visibility and information content of the interface of the developed system allows its implementation even by users with average qualification [13].

We note that the phased solution of problems by the system modules in the order in which they are presented above in the Figure 4., allows to achieve the goal: automation of the processing, analysis, and classification of IR images with obtaining an "expert" opinion on the object under study.

We consider the methodology for processing the source IR images in each of the blocks of modules in order.

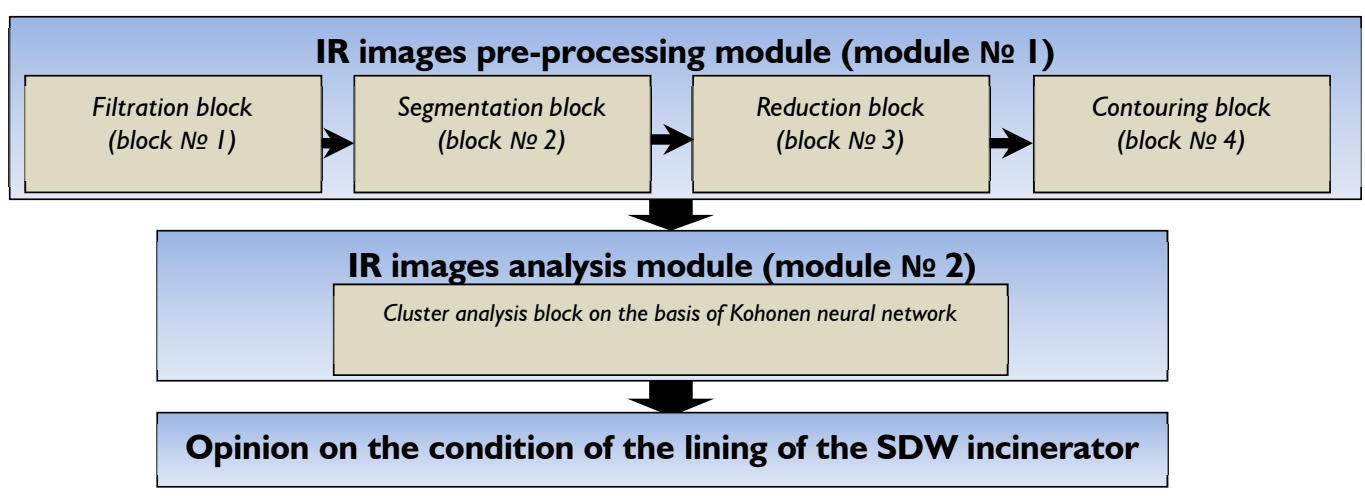

Figure 6. General scheme of functioning of the software system for processing and analysis of IR images 
The main problems in the implementation of image processing algorithms are limited amount of Random Access Memory (RAM), and the requirement to minimize the processing time of large volumes of information. In this connection, an important characteristic of image processing operations is the possibility of their decomposition into simpler, elementary operations on a limited number of elements of the original image. Therefore, all the IR image processing methods described below implemented in the corresponding functional blocks, were based on local or element wise operations.

In this Filtration block, median filtration is used, which allows eliminating various artifacts in the image in the form of low-frequency noise caused by defects in the lens of the thermographic camera, dust

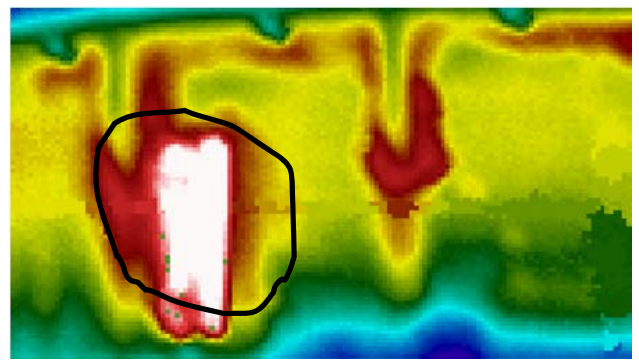

a) suspensions in the atmosphere, and errors in the digitization of the analog signal, and, thus, improves image quality. It is implemented by moving a certain mask (in our case, $3 \times 3$ ) along a discrete image and replacing the image value in the center of the mask with the median value of the original samples inside the aperture. As a result, we obtain a smoother image than the original one. The local type of the filtration operation is used. An example of the initial and filtered image of the lining is shown in the Figure 7, $\mathrm{a}$ and $\mathrm{b}$ respectively. It is easy to note, that in the area surrounded by the curve, after filtering, artifacts in the form of green dots practically disappeared, which could be due to ordinary dust suspensions in the atmosphere or defects in the thermal imager lens.

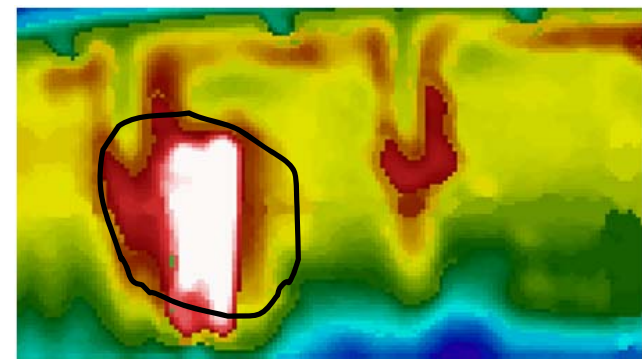

b)

Figure 7. Unfiltered (a) and filtered (b) IR image of furnace linings

The method of threshold segmentation in the Segmentation block is used in this block, which allows removing part of the redundant information from the image and distinguishing essential information from it. The segmentation method is the global threshold. The type of performed operation is element-wise. As can be seen from the Figure 8 (b), two global thresholds were selected, as a result of which only three colors remained on the segmented

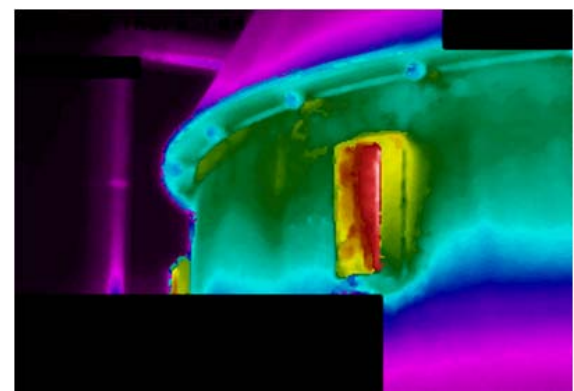

a) images: "green - the area of the furnace lining without damage", "yellow - the area of increased wear of the furnace lining", "red - the area of burnout or extreme wear of the furnace lining (or the area of the furnace "window").

The threshold values were selected empirically, as well as using a brightness histogram showing the probability distribution of the appearance of pixels of one or another brightness in the image.

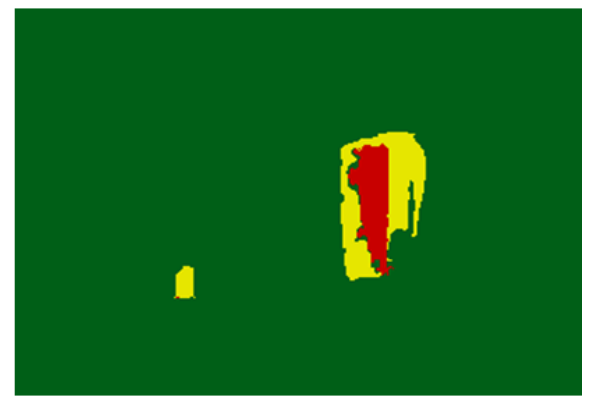

b)

Figure 8. Unsegmented (a) and segmented (b) IR images of the furnace lining

In the Reduction block, the pyramidal method of presenting images is used, which, like the threshold segmentation method, allows removing the redundant information from the image by reducing its size, while retaining the relative areas of informative regions. Due to this, it is possible to implement the analysis methods directly to the reduced image without any advance preparation. This accelerates the process of its further processing and analysis.

In the case under consideration, a two-layer pyramid was used, due to which the original images were reduced by 4 times. The type of operation is local. Figure 9 shows the initial segmented and reduced IR images. 


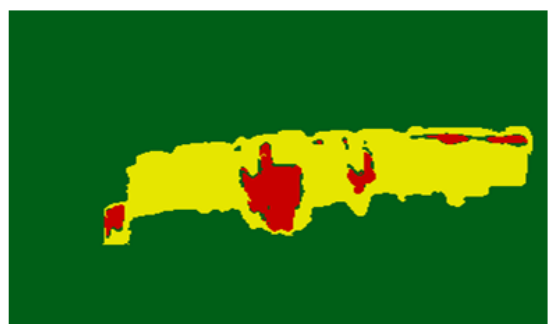

a)

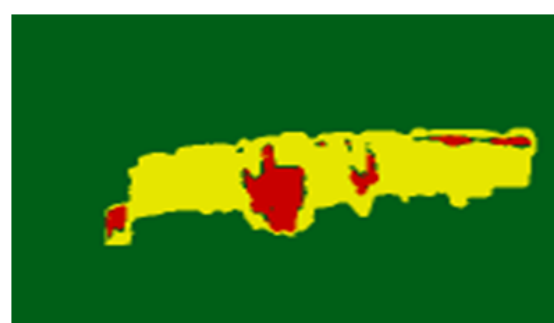

b)

Figure 9. The initial segmented (a) and reduced by 4 times IR image (b) of the furnace lining

To contour the informative regions in the image in Contouring block, the tracking algorithm of the "bug" is used. The advantage of this algorithm is its simplicity.

When applying this algorithm to IR images, information about each found area (separately for the red and yellow regions) is entered into a special structure, namely: coordinates of the points $(\mathrm{X}, \mathrm{Y})$ of the area boundary; absolute () in pixels) and relative

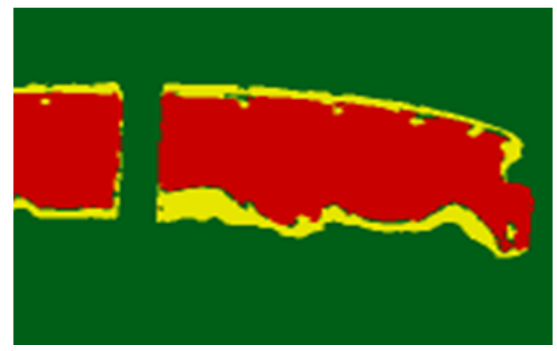

a) areas; number of the image, to which this area belongs. The index of the array of such structures denotes the area number in the image.

Contouring of the red and yellow areas was carried out separately. An example of selecting contours in an image is shown in the Figure 10. The work of this unit is the final step-by-step process of preliminary processing of IR images.

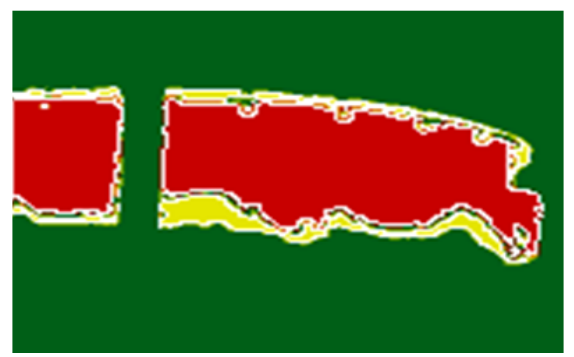

b)

Figure 10. IR image of the furnace lining without (a) contours and with red and yellow areas (b)

After consistent application of all blocks of the pre-processing module, we obtain reduced image, sufficiently "cleared" of noise, containing only informative regions; and the coordinates of the boundaries of each region are stored in a separate database. All this allows conducting the following cluster analysis with minimum time and maximum accuracy.

When analyzing and classifying the processed IR images by means of neural network technologies, the Kohonen artificial neural network was used in block "Cluster analysis" due to its invariance to rotations, movements and changes in the forms of informative regions, and also due to ability to train the network without a trainer when the required results are unknown in advance [14].

The structure of the Kohonen network used in cluster analysis in this work is shown in the Figure 11, where:

1) $x_{i}$ is the $i$ neuron of the input network layer $(i=$ $1, \ldots, 19200)$

2) $y_{j}$ is the $j$ neuron of the output network layer (cluster map) $(j=1, \ldots, 5)$;
3) $w_{i, j}$ is the connection strength between the $j$ neuron of the output layer and the $i$ neuron of the input layer.

The input value of a cluster map neuron is calculated by the following formula:

$$
u_{j}=\sum_{i} w_{i j} x_{i},
$$

where $u_{j}$ is the input value of a cluster map $j$ neuron. As it is shown in the formula (3), even a large number of links in a given network will not lead to a significant increase in the processing time of input data due to the use of linear combination of inputs to calculate the input value of a cluster map neuron [15]. In our system, 5 neurons were used in the output layer of the Kohonen network (cluster map). The dimension of the input network layer corresponded to the size of the processed image (160x120=19200 neurons). Thus, the total number of connections in the network was $19200 * 5=96000$.

The input data vector for accelerating the network training is preliminarily normalized by the following formula: 


$$
\frac{x}{\sqrt{\sum_{i} x_{i}^{2}}} \rightarrow X
$$

where $x_{i}$ is the $i$ component of the input vector $(i$ pixel of the image), $x$ is the input vector, $\mathrm{X}$ is the normalized input vector. Normalization of input data has a positive impact on the speed of network training. Before normalization, the input value for the red pixel of the IR image was 1 , the input value for the yellow one was 0.5 , and the input value for the green one ("fine", see below) was (-0.08).

In addition to normalizing the input vector, to accelerate the network training, the initial weights of the neurons were initialized not by random values, but by the same value obtained from the conditions of the convex combination [16]: $w_{i j}=\frac{1}{\sqrt{n}}$, where $n$ is the number of neurons in the output network layer $(n=5)$.

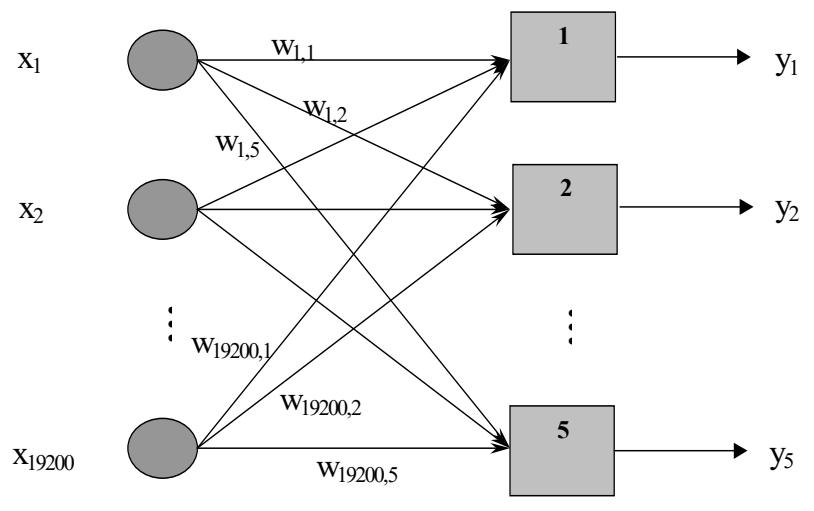

Figure 11. Structure of the Kohonen network used in the program

The SOFTMAX function was used as the activation functions of neurons of the output layer [17]:

$$
Y_{j}=\frac{e^{u_{j}}}{\sum_{k} e^{u_{k}}},
$$

where $Y_{j}$ is the output of the $j$ neuron, $u_{j}$ is the weighted sum of inputs of the $j$ neuron; the sum is taken for all neurons in the output network layer $(\mathrm{k}=1, . ., 5)$. Due to this, the level of activation of the output layer neurons can be considered as the probability of belonging of the entire original image or its particular region to a certain class (cluster) [18].

When training the network, the technology of winning neurons inhibition is used, based on a decrease in the value of weight correction with an increase in the number of winnings of these neurons in order to avoid the saturation effect and put other neurons of the cluster map into operation. In this case, the weight coefficients of the winning $\mathrm{k}$ neuron are corrected by the following formula:

$$
w_{i k}=w_{i k}+\alpha_{k}(t) \cdot\left(x_{i}-w_{i k}\right)
$$

where $w_{i k}$ is the weight coefficient of the $i$ input of the winning neuron; $x_{i}$ is the value of the winning neuron at the $i$ input; $\alpha_{k}(t)$ is the training speed for the $k$ neuron. The learning speed can be either a small numeric constant or a certain function, which steadily decreases during the network training process. Thus, it appears that the more often a neuron wins, the less and less its weights change, i.e. inhibition and fixation of the weights is carried out [19]. Algorithmically, the time course in neural network is determined by iterative execution of the same actions on neurons.

The following function was selected as a steadily decreasing function simulating the network training speed:

$$
\alpha_{k}(t)=0.4(1-\tanh (z(t))),
$$

where $z(t)=L(t) / M$ is the function that steadily increases from a certain point in time $t$, and takes into account how many times in a row the $k$ neuron won in the same set of marked IR images. In the function $\mathrm{z}(\mathrm{t}), \quad \mathrm{M}$ is a certain numeric constant found experimentally, which controls the speed of decrease of $\alpha_{k}(t)$, as the quality of recognition increases; $\mathrm{L}(\mathrm{t})$ is a parameter that determines how many times in a row the network was able to correctly classify the input vector (the correct classification is understood as the winning of the same neuron for input images belonging to the same class). If a neuron "won" on an input image, which does not belong to a neuron class, then the number of correct neuron classifications is reset $(L(t)=0)$, and a neuron class is reoriented to the class of the given input image. Network training stops after the number of consecutive successful classifications exceeds a certain number for all neurons (or only for selective ones, depending on the task being solved). Number 10 was chosen for artificial neural network in this system, i.e. the training stops when $L(t) \geq 10$ for each neuron from the cluster map of the network (Figure 12.). Such approach accelerates the network training process and allows smooth adjusting of its training speed. The value of 0.4 was chosen experimentally as the initial value of the function $\alpha(t)$ for all neurons of the output layer of the artificial neural network.

In the process of training of the Kohonen network, a "fine" was also used for processing non-essential ("green") pixels of the IR image. The use of a "fine" is necessary for the reduction of the response of neurons that have already won enough times. The value of "fine" was selected experimentally in the process of the network training on various examples; and the "fine value" for the green pixels was chosen in such a way that the analysis conducted by the network was more "pessimistic" in order to "reinsure" in making decisions about the repair of the lining [1].

Such approach is due to the fact that the winning neurons have greater values of weight connections than neurons that either never won, or very rarely 
won. Due to the negative value of activation of the input neurons for the green pixels, it is possible to reduce the value of activation of the output neurons with a larger number of wins, and thus to involve other neurons in the clustering process [20].

A well-trained network should provide the maximum value on the same neuron of the output layer for similar input vectors. After training and identifying the clusters in the output layer, they are usually analyzed and given meaningful values according to the area of use of the initial data and the conditions of the set task.

\section{Results of the Program on the Example of Processing and Analysis of a Certain Set of IR Images}

The result of training of the Kohonen network is shown in the Figure 12. There was no uniformity in the distribution by the number of critical regions on the training IR images, i.e. the training set was compiled in a rather arbitrary manner, only with compliance with certain formalities.

Frequencies of win of each neuron in the Kohonen network in processing images of the incinerator lining as a whole

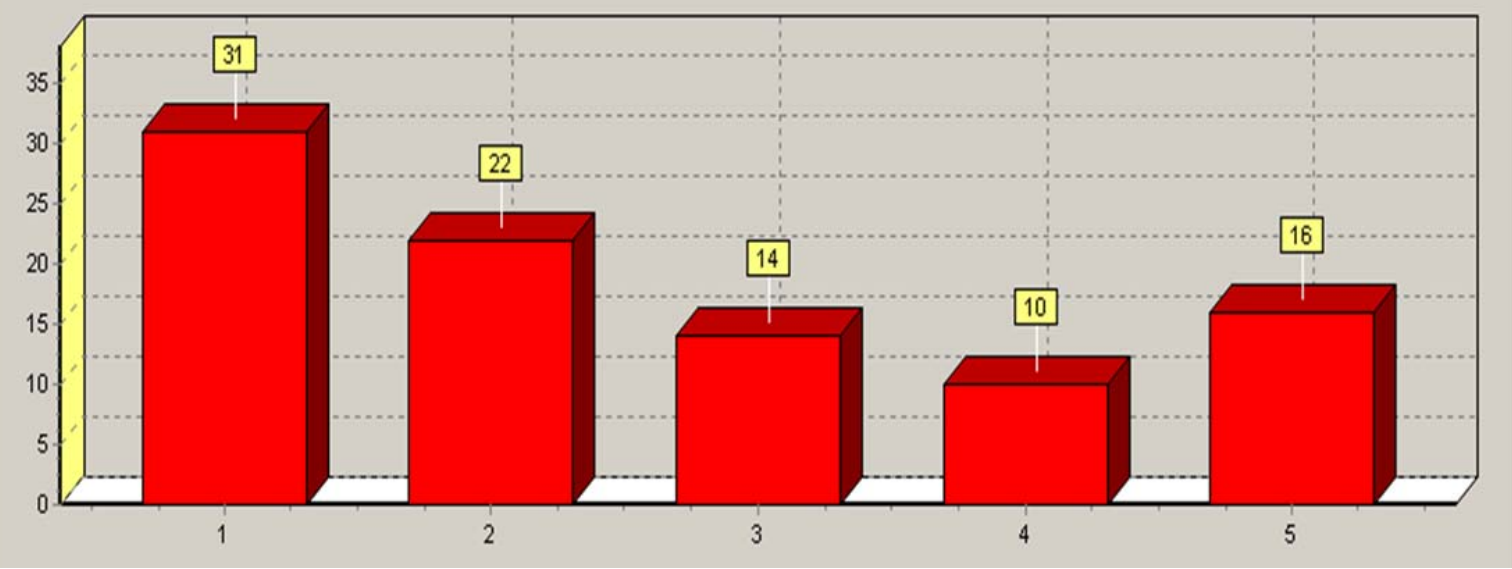

Figure 12. Result of the Kohonen network training on IR images of the incinerator lining

However, this fact affects the quality of network training and the results of the analysis of test images, which is another advantage of cluster analysis on the basis of the Kohonen network.

In the graphs in the Figure 8., the vertical axis shows the number of "successful" classifications for a neuron (numbers above the columns) that happened consecutively (within the same epoch), i.e. when a neuron "won" on the same images in the process of the network training. The horizontal axis shows the numbers of neurons of the output layer of the Kohonen network (cluster map).

The example of cluster analysis is shown in the Figure 13. The Figure 14 shows images № 19 and № 20 , which were used as test cases for testing the trained Kohonen network.

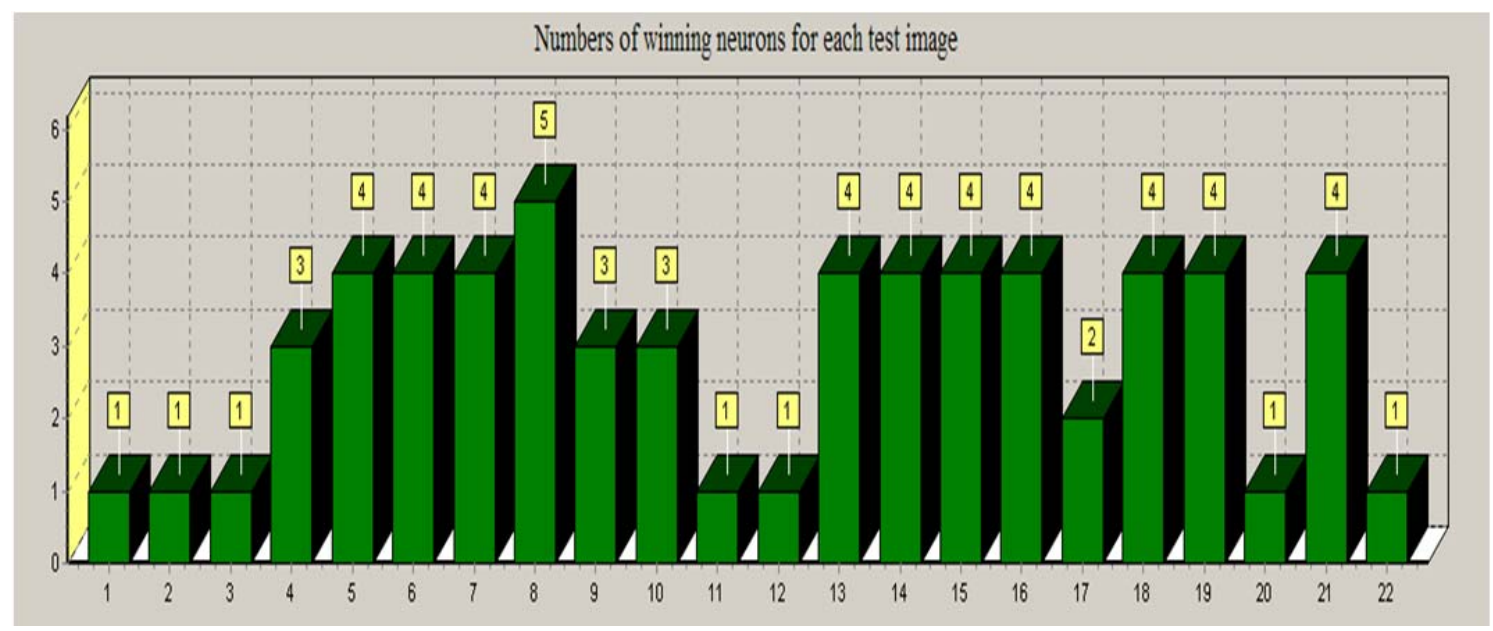

Figure 13. Result of cluster analysis of test images of the incinerator lining as a whole 
In the graphs presented in the Figure 13, the vertical axis shows the number of the cluster map of the Kohonen network (numbers above the columns

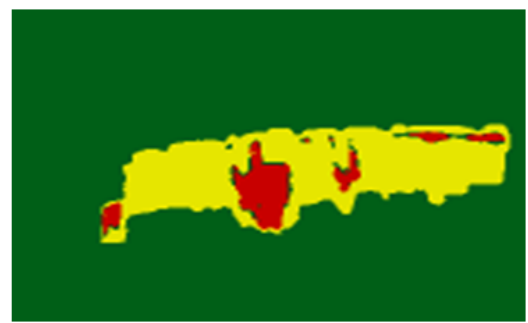

a)

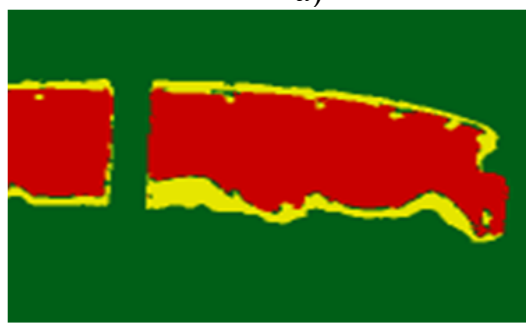

show the number of the "winning" neuron for a certain image). The horizontal axis shows the numbers of test images.

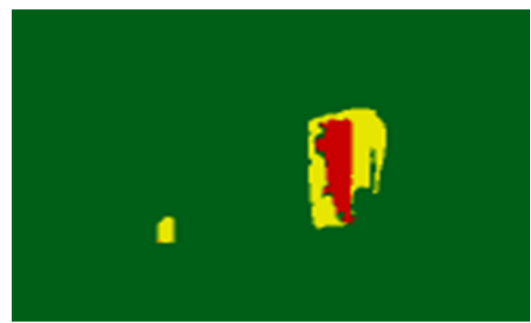

b)

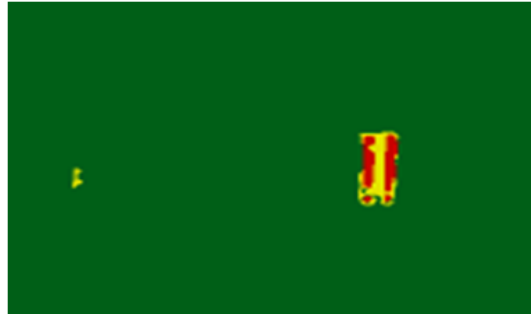

Figure 14. Test images № 19 (a), 20 (b), 21 (c), and 22 (d)

As it is shown in the Figure 13. and Figure 14., the images belong to different classes: image № 19 belongs to class 4, image № 20 belongs to class 1 , image № 21 belongs to class 4, and image № 22 belongs to class 1 . On the basis of the data obtained for each training image during the experimental study, each neuron-cluster in the Kohonen network received meaningful information carrying substantive basis about the object of study (the number of the most activated neuron corresponds to the class number):

a) neuron № 1: the incinerator lining is in good condition. Repair is not required;

b) neurons № 2 and 3: the incinerator lining in this place should be under control. Repair as necessary;

c) neurons № 4 and 5: the incinerator lining in this place requires major repairs.

Returning to the Figure 14, we can see that the obtained clustering corresponds to the facts. However, two interesting points should be made:

- first, the images № 19 and 21 belong to the same damage class, i.e. the network "decided" that the wear of the lining is moderate, but over a sufficiently large area it is equal to the requirement to replace the incinerator lining under intense wear, which allows taking preventive measures on ensuring the safe functioning of the incinerator;

- second, the images № 20 and 22 belong to class 1 , as in this case, the red areas in the images correspond to the technological "windows" of the SDW incinerator which are clearly visible in the Figure 1 and stand out against the temperature background compared to the entire lining. These areas are not damaged, and the neural network safely identifies them with class 1 when repair is not required. So, the "red" sections of a small area of the artificial neural network are identified with the technological "windows" in the SDW, and not burnout areas, which is quite correct. Typically, the furnace lining burns out in large areas, and the presence of a locally destroyed area is unlikely.

\section{Conclusions}

On the basis of the considered technology of automated IR image processing and analysis using a modular computing system, the conclusion can be drawn that the methods described above are a powerful tool in working with IR images, which allows the following:

a) excluding the expert from the chain of image processing and analysis, and thus reducing the degree of subjectivity in decisions on the lining repair;

b) significant reducing of workload of an experts in processing large volumes of data, and thus eliminating the probability of erroneous conclusions due to their fatigue. At the same time, a system configured by qualified specialists according to the studied objects and data area will constantly demonstrate high-quality results, regardless of time and place of work;

c) modularity of the software system design allows, if necessary, modifying one or another functional block with no difficulty, according to the latest advances in digital processing and image analysis, without affecting other blocks;

d) this system is much more productive compared to a person, especially in analyzing a large number of IR images due to human fatigue caused by intensive work for a long period of time, and also due to modern advances in computer hardware and software. 


\section{References}

[1].Tarasov, V. V., Torshina, I. P., \& Yakushenkov, Yu. G. (2014). Modern problems of optoelectronics: study guide. Moscow: Moscow State University of Geodesy and Cartography (in Russ.).

[2].Rannev, G. G. (2007). Izmeritel'nyye informatsionnyye sistemy. Moscow: Publishing House of Moscow State Regional University (in Russ.).

[3].Lloyd, J. M. (2013). Thermal imaging systems. Springer Science \& Business Media.

[4].Milovzorov, V. P. (1989). Elements of information systems. Moscow: High School (in Russ.).

[5].Bramson, M. A. (1964). Infrared radiation of heated bodies. Moscow, Nauka, 233.

[6].Miroshnikov, M. M. (2010). Teoreticheskie osnovy optiko-jelektronnyh priborov (Theoretical Foundations of optoelectronic devices). Moscow, Lan'[In Russian].

[7].V. P. Vavilov, Infrared Thermography and Thermal Control (Spektr, Moscow, 2009) [in Russian].

[8].Grishentsev, A. Y., \& Korobeynikov, A. G. (2014). Methods and Models of Digital Image Processing. St. Petersburg: Published in Politechnical University.

[9].Kruglov, V. V., \& Borisov, V. V. (2001). Artificial neural networks: Theory and practice. Goryachaya Liniya Telekom, Moscow.

[10]. Orr, G. B., \& Müller, K. R. (Eds.). (2003). Neural networks: tricks of the trade. Springer.
[11]. Gashnikov, M. V., Glumov, N. I., Ilyasova, N. Yu., Myasnikov V.V., Popov S.B., Sergeev V.V., Soifer V.A., Khramov A.G., Chernov A. V., Chernov V.M., Chicheva M.A., \& Fursov V.A. (2003). Computer image processing methods. Moscow: Physical and mathematical and technical literature (in Russ.).

[12]. Arkhangelsky, A. Ya. (2003). Programming in $C++$ Builder. Moscow: Binom-Press (in Russ.).

[13]. Stroustrup, B. (1994). The design and evolution of $\mathrm{C}++$. Pearson Education India.

[14]. Kallan, R. (2003). Osnovnye kontseptsii neironnykh setei. Neural Networks: Major Concepts), Moscow: Vil'yams.

[15]. Haykin, S. (2008). Neural Networks and Learning Machines (2rd Edition). Moscow: Williams (in Russ.).

[16]. Galushkin, A. I. (2012). Neural networks. Fundamentals of the theory. Hot Line-Telecom, 496.

[17]. Nikolenko, S., Kadurin, A., \& Arkhangelskaya, E. (2017). Deep learning. St. Petersburg.: Piter (in Russ.).

[18]. Kohonen, T. (2008). Self-organizing maps. Moscow: Binom, Knowledge Laboratory (in Russ.).

[19]. Samarasinghe, S. (2016). Neural networks for applied sciences and engineering: from fundamentals to complex pattern recognition. Crc Press.

[20]. Vantsov, S. V., Vasilyev, F. V., Medvedev, A. M., \& Khomutskaya, O. V. (2019). Epoxy-glass composite materials for substrate printed circuit boards gigabit electronics. Amazonia Investiga, 8(22), 434-442. 